\title{
Correction to: Polymer composites filled with core- shell structured nanofillers: effects of shell thickness on dielectric and thermal properties of composites
}

Dan Cao ${ }^{1}$, Wenying Zhou ${ }^{1, *}$ (1), Mengxue Yuan ${ }^{2, \star}$, Bo Li ${ }^{3}$, Ting $\mathrm{Li}^{1}$, Jin $\mathrm{Li}^{1}$, Dengfeng Liu ${ }^{1}$, Guangheng Wang ${ }^{1}$, Juanjuan Zhou ${ }^{1}$, and Hongfang Zhang ${ }^{4, *}$

${ }^{1}$ School of Chemistry and Chemical Engineering, Xi'an University of Science \& Technology, Xi'an 710054, China

${ }^{2}$ Materials Research Institute, The Pennsylvania State University, University Park, PA 16802, USA

${ }^{3}$ PolyK Technologies, State College, PA 16803, USA

${ }^{4}$ School of Physical Science and Technology, Suzhou University of Science and Technology, Suzhou 215009, China

Published online:

3 February 2022

(C) Springer Science+Business Media, LLC, part of Springer Nature 2022

Correction to: Journal of Materials Science: Materials in Electronics (2022) https:/doi.org/10.1007/s10854-022-07705-z

Figure 3 in the original article has been found to be erroneous after checking the EDS raw data. Provided below is the correct version of Fig. 3. This correction does not affect the conclusion.

The original article can be found online at https:/ / doi.org/10.1007/s10854-022-07705-z.

Address correspondence to E-mail: wyzhou2004@163.com; muy151@psu.edu; constance_zhanghf@126.com 

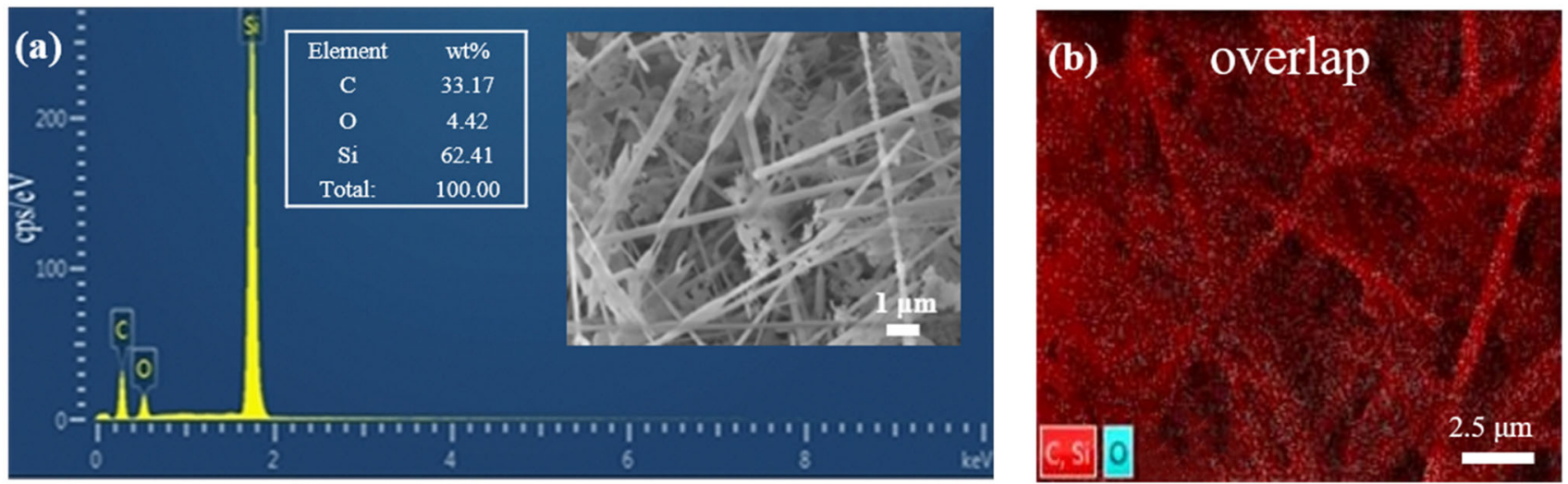

(c)
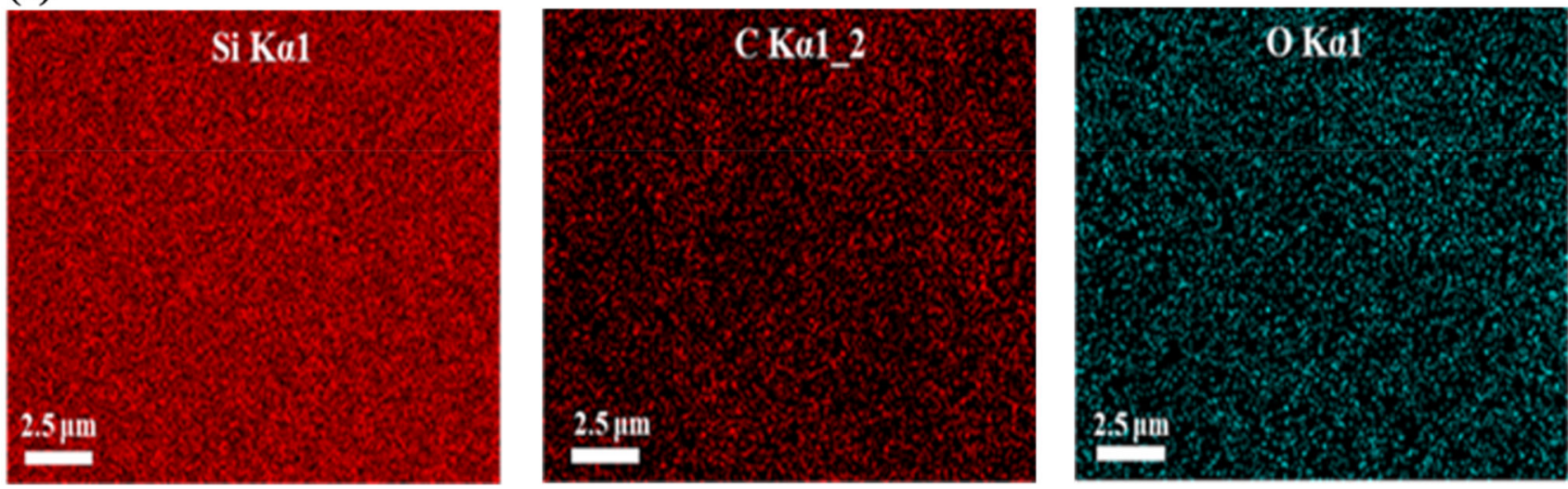

Fig. 3 a EDS spectrum, the inset standing for the SEM image of $\beta-\mathrm{SiC}_{\mathrm{w}} @ \mathrm{SiO}_{2}, \mathbf{b}-\mathbf{c}$ the corresponding element mapping for $\beta$ $\mathrm{SiC}_{\mathrm{w}} @ \mathrm{SiO}_{2}$

Publisher's Note Springer Nature remains neutral with regard to jurisdictional claims in published maps and institutional affiliations. 